\author{
Jean Michel Lemoyne de Forges \\ Professeur émérite de l'Université Panthéon-Assas, Paris-2
}

\title{
LA DÉCONCENTRATION ADMINISTRATIVE
}

\author{
UDK: 342.9 \\ Primljeno: 1. rujna 2018. \\ Izvorni znanstveni rad
}

L'établissement d'un État moderne en Europe a entraîné l'apparition d'un système d'introduction d'un État sur l'ensemble de son territoire. La raison en est que les décisions gouvernementales doivent être appliquées de manière égale à tous. Cela ne peut être garanti que si les fonctionnaires, nommés par le gouvernement et responsables de la mise en œuvre et du suivi de la politique gouvernementale, les transfèrent effectivement du niveau de fonctionnaire gouvernemental au niveau local et régional. En règle générale, l'administration centrale s'acquitte de tâches de caractère national dont la mise en œuvre en vertu de la loi ne peut être confiée à un niveau régional. Cependant, l'État doit également avoir des fonctionnaires au niveau local qui mettront en œuvre de véritables pouvoirs lors de la prise de décisions (sur la base de la délégation de pouvoirs), afin de prendre en compte les besoins et la situation locales. La «déconcentration» représente précisément cela. Par conséquent, nous traitons avec des services qui incluent des fonctionnaires nommés qui sont soumis à l'autorité d'un organisme central et qui représentent localement le gouvernement et les ministères. En France et en général, nous distinguons trois catégories d'administrations: l'administration centrale, l'administration périphérique (décentralisée ou déconcentrée), l'administration spécialisée indépendante qui reste néanmoins rattachée à l'un des ministères compétents qui supervisent les organismes placés sous le contrôle du gouvernement. La déconcentration à la française enrichit la dimension interministérielle, présente localement chez le préfet qui représente le président du gouvernement et les ministres et qui est chargé de la gestion du territoire, du dialogue avec les représentants locaux du pouvoir exécutif et de la modernisation de l'administration. Compte tenu de la carte administrative de la France comprenant trente mille communes, une centaine de départements et une vingtaine de régions (13 depuis 2015), la déconcentration repose sur les éléments suivants: le préfet de région met en œuvre la politique nationale et la politique de la communauté (européenne) fixe des objectifs stratégiques, alloue des ressources, évalue l'activité de l'Etat. Le préfet de département est responsable de l'activité opérationnelle et de l'administration des politiques publiques. Le vice-préfet initie et rejoint des partenaires dans des «centres de vie» (villes et villages) au sein d'un département.

\section{Mots clés: administration, déconcantation, Europe, France}

Pour résumer l'expérience française de déconcentration administrative, il suffit de lire le début du décret $\mathrm{n}^{\circ}$ 2015-510 du 7 mai 2015 dont le titre exact est « décret portant charte de la déconcentration ». Ce titre, repris d'un décret de 1992 ayant le même objet, est destiné à montrer qu'il s'agit de poser les principes de l'organisation des services de l'État, et seulement de l'État, pas du tout des autres personnes publiques, collectivités locales ou établissements publics, notamment. En outre, le chapitre $1^{\mathrm{er}}$ de ce décret porte sur les « règles d'attribution des administrations 
civiles de l'État ». Il s'agit donc clairement de l'organisation des services non militaires de l'État.

Article $1^{\text {er }}$ : « La déconcentration consiste à confier aux échelons territoriaux des administrations civiles de l'État les moyens et la capacité d'initiative pour animer, coordonner et mettre en œuvre les politiques publiques définies au niveau national et européen, dans un objectif d'efficience, de modernisation, de simplification, d'équité des territoires et de proximité avec les usagers et les acteurs locaux »

Cette définition, fruit d'une longue expérience administrative depuis le $\mathrm{XIX}^{\circ}$ siècle, montre à la fois que la déconcentration est un mode d'organisation administrative de l'État et qu'elle a une finalité managériale. Le coeur de ces deux dimensions, organisationnelle et managériale, c'est le territoire : «On peut gouverner de loin mais on n'administre que de près » (Persigny, Ministre de l'Intérieur, en 1852).

Traditionnellement on oppose la déconcentration à la décentralisation : l'administration d'un territoire donné, par exemple celui d'une ville, peut être confiée soit à des administrateurs indépendants de l'État, désignés par les habitants de ce territoire et ne rendant compte de leur gestion qu'à ces électeurs (c'est le principe de la décentralisation), soit à des administrateurs désignés par le gouvernement de l'État, placés sous l'autorité hiérarchique de ce gouvernement et ne rendant compte de leur gestion qu'à ce gouvernement (c'est le principe de la déconcentration).

L'aspect territorial rapproche ces deux modèles d'organisation administrative. La tradition française l'exprime bien puisque, depuis la fin du XIX ${ }^{\circ}$ siècle, si le territoire du « département » est le support géographique d'une collectivité locale administrée par des organes élus par la population, il est en même temps le cadre géographique d'un ensemble de services de l'Etat placés sous l'autorité d'un représentant du gouvernement, le préfet. Et, lorsqu'un cadre géographique plus vaste, la région, s'est progressivement imposé comme étant plus rationnel pour certaines fonctions administratives, le même modèle a été reproduit : le cadre géographique de la région sert en même temps de support à une collectivité territoriale dotée de ses propres organes élus et de cadre à un certain nombre de services administratifs de l'État dirigés par un préfet de région.

Cette territorialisation de l'action de l'État présente les avantages de la proximité ; elle permet donc d'adapter la mise en oeuvre des politiques publiques de l'État aux réalités locales ; en outre, lorsque le cadre géographique retenu est le même pour les collectivités décentralisées et pour les services déconcentrés de l'État, il est plus facile de coordonner l'action de l'État avec celle de la collectivité locale, voire de conduire des politiques publiques locales conjointes.

Le principe de la déconcentration est que chaque ministère dispose, dans les régions ou dans les départements, de services administratifs qui lui sont rattachés ; la mise en œuvre de la déconcentration des services de l'État pose donc trois types de problèmes : 
- le premier est de répartir les fonctions entre les services centraux (les ministères) et les services déconcentrés ;

- le deuxième est d'organiser les relations entre les ministères et les services déconcentrés ;

- le troisième est d'assurer la cohérence de l'action de l'État dans les services déconcentrés.

\section{I - LA RÉPARTITION DES ATTRIBUTIONS}

L'article $1^{\text {er }}$ alinéa 2 du décret du 7 mai 2015 pose le principe selon lequel la déconcentration « constitue la règle générale de répartition des attributions et des moyens entre les échelons centraux et territoriaux des administrations civiles de l'Etat »; l'article 2 alinéas 3 et 4 du même décret ajoutent : "Sont confiées aux administrations centrales et aux services à compétence nationale les seules missions qui présentent un caractère national ou dont l'exécution, en vertu de la loi, ne peut être déléguée à un échelon territorial ./ Les autres missions, notamment celles qui intéressent les relations entre l'État et les collectivités territoriales, sont confiées aux services déconcentrés»; de son côté, l'article 3 - I précise : «Les administrations centrales assurent, au niveau national, un rôle de conception, d'animation, d'appui des services déconcentrés, d'orientation, d'évaluation et de contrôle./ A cette fin, elles participent à l'élaboration des projets de loi et de décret et préparent et mettent en œuvre les décisions du Gouvernement et de chacun des ministres(...) »; enfin la suite de cet article énumère les principaux domaines dans lesquels s'exercent ces missions des administrations centrales, en commençant par « la définition et le financement des politiques nationales, le contrôle de leur application (notamment par les services déconcentrés), l'évaluation de leurs effets ».

On le voit, aux termes de la charte de la déconcentration, pour l'exécution des politiques publiques de l'État, le principe est la compétence des services déconcentrés, les services centraux n'ayant qu'une fonction de conception, d'orientation et de contrôle. Ainsi, le principe de subsidiarité, qui a toujours plus ou moins inspiré la décentralisation, est ici d'une certaine manière appliqué aussi à la déconcentration. Ceci est destiné à clarifier la place des services déconcentrés et à mettre fin à un certain désordre dans leurs relations avec les ministères (V. ci-dessous).

Ce mécanisme de limitation des compétences des services centraux de l'État appelle deux compléments.

En premier lieu, les ministères et leurs services déconcentrés n'ont pas le monopole de la mise en œuvre des politiques publiques nationales. L'État a depuis longtemps créé des «établissements publics nationaux », personnes morales de droit public chargées, sous le contrôle de l'Etat, de l'élaboration et de l'exécution de certaines politiques publiques (Office national des anciens combattants et des victimes de guerre, Centre des monuments nationaux...). Aujourd'hui beaucoup de ces établissements publics ont pris le nom d'agence (Agence nationale de 
l'habitat...), tandis que d'autres agences d'exécution (executive agencies), aux statuts juridiques les plus divers ont été créées au niveau national ou parfois au niveau régional (Agences régionales de santé). Dans la mesure où certains de ces organismes disposent eux-mêmes de services déconcentrés, la charte de la déconcentration de 2015 a tenu à préciser que la déconcentration « implique l'action coordonnée de l'ensemble des services déconcentrés et des services territoriaux des établissements publics de l'Etat » (art. 1 ${ }^{\mathrm{er}}$, al. 3).

En second lieu, il est évidemment nécessaire de préciser quels sont les échelons territoriaux des services déconcentrés des ministères, et d'organiser la répartition des attributions entre ces différents échelons. A cet effet, la charte de la déconcentration mentionne trois circonscriptions territoriales possibles pour les administrations civiles de l'État, la « circonscription régionale », la « circonscription départementale » et « l'arrondissement » (subdivision traditionnelle de la circonscription départementale). La charte pose en outre le principe général selon lequel « la circonscription départementale est l'échelon territorial de mise en œuvre des politiques nationales et de l'Union européenne », tandis que les missions relevant de la circonscription régionale, bien qu'assez larges, sont limitativement énumérées (animation et coordination des politiques de l'État, mise en œuvre des politiques nationales et européenne en matière d'emploi, d'innovation, de culture, de statistiques publiques, de développement économique et social et d'aménagement durable du territoire, coordination des actions intéressant plusieurs départements de la région, modernisation des services déconcentrés, définition de la stratégie immobilière de ces services déconcentrés, programmation et répartition des crédits de l'État, contractualisation des programmes pluriannuels conjoints de l'État et des collectivités locales) (art. 4). On remarquera le soin avec lequel le vocabulaire est choisi, en particulier pour bien distinguer l'exécution proprement dite des politiques publiques ( " mise en oeuvre ») des activités de conception, d'animation et de coordination des missions dont l'exécution relève d'autres entités.

Pour en terminer avec ce premier point, il convient d'insister sur le fait que la charte de la déconcentration n'est qu'un décret qui pose des principes d'organisation. A plusieurs reprises elle souligne que la loi - et même d'autres décrets - peuvent prévoir une répartition différentes des compétences dans certaines matières.

\section{2 - LES RELATIONS ENTRE ADMINISTRATIONS CENTRALES ET SERVICES DÉCONCENTRÉS}

Si les services déconcentrés sont ainsi dotés d'attributions administratives propres, sur lesquelles, normalement, ne peuvent empiéter les services centraux des ministères, il est clair que les ministres sont les supérieurs hiérarchiques de tous les services administratifs de l'État, de sorte que ce sont bien les administrations centrales qui ont pour mission d'orienter, de contrôler et d'évaluer l'activité des services déconcentrés. 
C'est pourquoi, dans le fonctionnement administratif de l'État, divers instruments juridiques sont utilisés.

Tout d'abord, le principe même de la déconcentration suppose que les chefs des services déconcentrés disposent du pouvoir de prendre des décisions (ou de conclure des contrats) au nom de l'État. Ainsi, tantôt ils sont investis de telles compétences directement par la loi ou par un acte réglementaire pris au niveau national, ou bien les ministres leur donnent des délégations de pouvoir dans les matières relevant de leur compétence. En France, aux termes du décret n 2004-374 du 29 avril 2004 relatif aux pouvoirs des préfets ce sont les préfets (de région ou de département, selon la matière en cause) qui représentent les ministres et qui, en conséquence, peuvent seuls recevoir de leur part des délégations de pouvoir. Mais il n'en a pas toujours été ainsi : jadis les chefs des services déconcentrés (p. ex. les anciens directeurs départementaux de la santé) recevaient délégation de leur ministre de rattachement (p. ex. le ministre de la santé) ; il existe d'ailleurs encore aujourd'hui des exceptions notables (p. ex. les recteurs, représentants du ministre chargé de l'enseignement).

L'autre grand instrument juridique de mise en œuvre de la déconcentration, ce sont les circulaires, instructions, directives et autres « lignes directrices » élaborées par les administrations centrales (signées par le Premier ministre lui-même, par un ministre ou par un directeur d'administration centrale selon le cas); chaque année, plusieurs milliers de documents de ce type sont adressés aux services déconcentrés. Ces documents peuvent avoir deux fonctions principales : les uns («circulaires ») interprètent et explicitent le contenu des lois et règlements et expliquent aux fonctionnaires, souvent de manière très détaillée, la manière dont ils doivent les appliquer. Leur régime juridique est aujourd'hui bien encadré. Depuis un décret du 8 décembre 2008 si elles ne sont pas publiées sur le site internet du Premier ministre créé à cet effet (circulaires.legifrance.fr), elles ne sont pas applicables. En outre, lorsqu'une circulaire ne se borne pas à interpréter une réglementation existante mais contient des dispositions « de caractère impératif », elle peut faire l'objet d'un recours pour excès de pouvoir devant le juge administratif et être annulée, notamment si elle émane d'une autorité incompétente ou si elle n'est pas conforme à la réglementation qu'elle prétendent expliciter (C.E. sect. 18 déc. 2002, Mme Duvignères).

Pour leur part, ce que, depuis un arrêt Jousselin du 19 septembre 2014, la jurisprudence du Conseil d'État appelle aujourd'hui les « lignes directrices » concerne des domaines dans lesquels les autorités administratives sont investies d'un pouvoir discrétionnaire d'appréciation ; ces autorités, ou leurs supérieurs (tels, précisément, les ministres) peuvent élaborer des actes, qui doivent eux aussi être publiés, indiquant de quelle manière, et en particulier sur la base de quels critères d'appréciation, ce pouvoir discrétionnaire doit être exercé.

Enfin il faut noter que, grâce aux réseaux intranets des administrations, les relations entre les services déconcentrés et les administrations centrales sont plus souples, mais aussi moins visibles pour le public, malgré l'obligation de publication 
des circulaires et des « lignes directrices ». Ainsi une circulaire du Premier ministre du 17 juillet 2013 relative à la simplification administrative et aux relations avec les services déconcentrés a recommandé d'utiliser ces réseaux « pour la diffusion des recommandations, éclaircissements et précisions nécessaires à l'activité des préfets et des services déconcentrés », y compris « sous forme de questions-réponses ou de forums d'échanges ». Ainsi, si en principe les services centraux ne doivent pas traiter des dossiers particuliers qui relèvent de la compétence des services déconcentrés, de tels dialogues permettent de tourner facilement ce principe.

\section{3 - LA COHÉRENCE DES L'ACTION TERRITORIALE DE L'ÉTAT}

La nécessité de la déconcentration est une évidence dans tous les Etats d'une certaine superficie. Mais, outre le problème des relations entre chaque ministère et ses services déconcentrés, elle pose aussi celui de la cohérence de l'action territoriale de l'État. En effet, assez naturellement, les fonctionnaires de chaque ministère ont tendance à ignorer ce que font leurs collègues des autres ministères. Au niveau central, en principe, différents mécanismes de coordination sont destinés à garantir la cohérence du travail gouvernemental sous l'autorité du chef du gouvernement ; dès que l'on s'éloigne de la capitale, les effets de cette coordination interministérielle s'estompent.

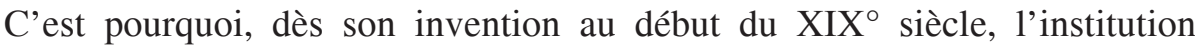
préfectorale a eu pour objet de garantir la cohérence de l'exécution, dans les départements, de la loi et des décisions gouvernementales ; l'article 3 de la loi du 28 pluviôse An VIII disposait déjà que, dans le département, « le préfet sera chargé seul de l'administration ».

Aujourd'hui, l'article $1^{\text {er }}$ du décret $n^{\circ}$ 2004-374 du 29 avril 2004 « relatif aux pouvoirs des préfets, à l'organisation et à l'action des services de l'État dans les régions et départements » (modifié notamment par un décret du 16 février 2010) dispose : « Le préfet de région dans la région, le préfet de département, dans le département, est dépositaire de l'autorité de l'État./ Ils ont la charge des intérêts nationaux et du respect des lois./ Ils représentent le Premier ministre et chacun des ministres./ Ils veillent à l'exécution des règlements et des décisions gouvernementales./ Ils dirigent, sous l'autorité des ministres et dans les conditions définies par le présent décret, les services déconcentrés des administrations civiles de l'Etat ».

Pour assurer la mise en œuvre de ces principes se sont développés les outils juridiques et administratifs destinés d'abord à organiser la coordination entre les services déconcentrés puis, progressivement, à assurer l'autorité des préfets sur ces services. Ils sont trop nombreux pour être tous analysés ici ; on ne citera que quelques exemples. 
Ainsi, un des plus anciens (1964) fut l'obligation, pour tous les chefs des services déconcentrés, de ne correspondre avec leur ministère de rattachement, que par l'intermédiaire du préfet ; cette règle simple a ensuite été étendue aux communications des chefs de ces services entre eux et avec les collectivités locales et leurs établissements publics .

On doit aussi citer les réunions périodiques obligatoires des chefs des services déconcentrés sous la présidence du préfet (comité d'administration régionale, collège des chefs de service), la règle selon laquelle seuls les préfets peuvent recevoir délégation de pouvoirs des ministres, à charge pour eux de consentir des délégations « de signature » aux chefs de service, la possibilité, pour les préfets, de réorganiser les services déconcentrés dans leur région ou dans leur département, la participation des préfets à la procédure de nomination des chefs de services et ensuite à leur évaluation professionnelle, ou encore les pouvoirs des préfets dans l'utilisation des crédits de l'État ... Tous ces procédés et bien d'autres (V. décret du 29 avril 2004) se sont surtout développés à partir de la politique de décentralisation conduite depuis 1982 : l'extension des compétences propres des collectivités territoriales s'est accompagnée d'un renforcement de la déconcentration, étant rappelé en outre que c'est le préfet qui exerce, au nom de l'État, le contrôle administratif des collectivités locales.

\section{CONCLUSION:}

Deux remarques pour conclure.

La première est que les préfets n'ont pas un monopole absolu de la direction des services déconcentrés des administrations civiles de l'État. Ainsi, depuis un décret de Napoléon de 1808 (17 mars), le ministre chargé de l'enseignement est aussi représenté par les recteurs d'académie compétent en matière de contenu de l'enseignement et de gestion des personnels. En matière financière, le principe de séparation des ordonnateurs et des comptables a conduit à interdire aux préfets de gérer la comptabilité publique (recettes et dépenses), celle-ci étant aujourd'hui confiée à un directeur régional des finances publiques qui relève directement du ministre des finances. Enfin, depuis 2009 (loi n 2009-879 du 21 juillet 2009), l'administration sanitaire et sociale déconcentrée est dirigée par les directeurs généraux des agences régionales de santé qui sont des subordonnés directs du ministre chargé de la santé (même si les A.R.S. sont des établissements publics).

La seconde est que, jusqu'à sa consécration par un décret de 1997, a progressivement émergé la notion de « service à compétence nationale». Il apparaît en effet que la mission de certains services dans les ministères exclut la possibilité de services déconcentrés dans les régions ou les départements. Ils sont alors labellisés « service à compétence nationale » et, quelle que soit leur appellation officielle (direction, service ou même agence), leur création et leur organisation obéissent à des règles particulières. C'est par exemple le cas des Archives nationales, important 

pour celui, tout aussi important, de l'organisation des administrations centrales.

\section{ADMINISTRATIVE DECONCENTRATION}

Establishing a modern state in Europe has brought about the appearance of a system of introducing state on all of its territory. The reason this has come about is the need for government decisions to be applied in an equal way to everyone. This can only be ensured if officials, whom the government appoints and who are responsible for implementing and following government policy, effectively transfer them from government official level onto local and regional level. Central administration generally singularly carries out tasks of a national character the implementation of which pursuant to law cannot be placed onto a regional level. However, the state also must have officials at a local level who will implement real powers when making decisions (based on delegating powers), in order to take care of local needs and circumstances. «Deconcentration» precisely represents that. Therefore, we are dealing with services which include appointed officials who are subject to central body authority and who locally represent the government and ministries. In France and in general, we differentiate among three categories of administration: central administration, peripheral administration (decentralized or deconcentrated), independent specialized administration which are nevertheless still linked to one of the relevant ministries which supervise the bodies which are under government control. Deconcentration French style enriches the inter-department dimension, locally present in the person named prefect who represents government president and ministers and who is in charge of managing territory, maintaining dialogue with local representatives of executive powers and modernization of administration. Given the administrative map of France which includes thirty thousand municipalities, about a hundred departments and twenty regions (13 since 2015), deconcentration is based on the following : regional prefect implements national policy and policy of (European) community establishes strategic goals, allocates resources, evaluates state activity. The department prefect is responsible for operative activity and administering public policies. The vice-prefect initiates and joins partners in so-called «life centers» (towns and villages) within a department framework.

Key words: administration, deconcantration, Europe, France

\section{UPRAVNA DEKONCENTRACIJA}

Uspostava moderne države u Europi dovela je do uvođenja države na cijelom njezinom teritoriju. Razlog za to je potreba da se njene odluke primjenjuju jednako na sve. To se može osigurati jedino ako službenici, koje vlada imenuje i koji su odgovorni za provedbu i praćenje vladine politike, iste učinkovito prenose s državne na lokalnu i regionalnu razinu. Središnja uprava općenito obavlja poslove nacionalnog karaktera čija se provedba sukladno zakonu ne može staviti na regionalnu razinu. Međutim, država također mora imati dužnosnike na lokalnoj razini koji će provoditi stvarne ovlasti prilikom donošenja odluka (na temelju delegiranja ovlasti), kako bi se brinuli o lokalnim potrebama i okolnostima. Ovdje je riječ o "dekoncentraciji". Radi se, dakle, o uslugama koje uključuju imenovane službenike koji podliježu središnjem tijelu vlasti i koji lokalno 
predstavljaju vladu i ministarstva. U Francuskoj i općenito razlikujemo tri kategorije uprave: središnja, periferna (decentralizirana ili dekoncentrirana), te neovisna specijalizirana uprava koja je ipak povezana s jednim od relevantnih ministarstava koja nadziru tijela koja su pod kontrolom vlade. Dekoncentracija na francuski način obogaćuje međuresorsku dimenziju, lokalno prisutnu u osobi imenovanog prefekta koja predstavlja predsjednika vlade i ministre i koja je zadužena za upravljanje teritorijem, održavanje dijaloga s lokalnim predstavnicima izvršne vlasti i modernizaciju uprave. S obzirom na administrativnu kartu Francuske koja uključuje trideset tisuća općina, oko stotinu odjela i dvadeset regija (13 od 2015.), dekoncentracija se temelji na sljedećem: regionalni župan provodi nacionalnu politiku i politiku (europske) zajednice uspostavlja strateške ciljeve, alocira resurse, ocjenjuje državnu aktivnost. Župan odjela je odgovoran za operativne aktivnosti i upravljanje javnim politikama. Potpredsjednik pokreće i pridružuje se partnerima u takozvanim «središtima života» (gradovima i selima) u okviru odjela.

Ključne riječi: uprava, dekonkantacija, Europa, Francuska 